\title{
Acute Responsive Elevation of Serum Secretory Acid Sphingomyelinase Activity in Infants with Respiratory Syncytial Virus Bronchiolitis
}

\section{Shoko Yamamoto}

Department of Pediatrics, Akita University Graduate School of Medicine

Atsuko Noguchi ( $\square$ atsuko@doc.med.akita-u.ac.jp )

Department of Pediatrics, Akita University Graduate School of Medicine

Chiaki Hatazawa

Division of Pediatrics, Akita Kousei Medical Center

\section{Takako Hatazawa}

Division of Pediatrics, Akita Kousei Medical Center

\section{Daiki Kondo}

Division of Pediatrics, Akita Kousei Medical Center

\section{Hiroki Kubota}

Division of Pediatrics, Akita Kousei Medical Center

\section{Satoko Tsuchida}

Division of Pediatrics, Akita Red Cross Hospital

\section{Tsutomu Takahashi}

Department of Pediatrics, Akita University Graduate School of Medicine

\section{Research Article}

Keywords: Respiratory syncytial virus, bronchiolitis, secretory acid sphingomyelinase

Posted Date: August 23rd, 2021

DOl: https://doi.org/10.21203/rs.3.rs-770495/v1

License: (c) (1) This work is licensed under a Creative Commons Attribution 4.0 International License. Read Full License 


\section{Abstract}

Background: Respiratory syncytial virus (RSV) bronchiolitis is the leading cause of hospitalization in infants. Biomarkers of disease severity may be significant in improving management. Blood secretory acid sphingomyelinase (S-ASM) activity has been recognized as a biomarker of cytokine release, inflammation, oxidative stress, and damage to the vascular endothelium in various diseases. The present study aimed to evaluate whether serum S-ASM activity is increased and correlates with disease severity in infants with RSV bronchiolitis.

Methods: Serum S-ASM activity was determined in 31 infants with RSV bronchiolitis, nine infants with RSV-negative febrile infection, and eight healthy infants. Laboratory data including two cytokines, interferon- $\gamma$ (IFN- $\gamma$ ) and interleukin-18, and clinical observatory findings, including clinical severity score specific for bronchiolitis, were analyzed for correlation with the levels of serum S-ASM activity.

Results: Serum S-ASM activity was significantly higher among the 31 infants with RSV bronchiolitis (9.5 $\pm 5.4 \mathrm{nmol} / \mathrm{mL} / \mathrm{h}$ ) than in those in each of the control groups (RSV-negative febrile infection patients, 4.0 $\pm 1.4 \mathrm{nmol} / \mathrm{mL} / \mathrm{h}, \mathrm{p}<0.005$; healthy controls, $4.3 \pm 1.9 \mathrm{nmol} / \mathrm{mL} / \mathrm{h}, p<0.005)$. Serum S-ASM activity negatively correlated with IFN- $y$ levels ( $r h o=-0.448, p=0.0129$ ), but were not correlated with any other outcomes analyzed in this study.

Conclusion: Serum S-ASM activity was significantly higher in infants with RSV bronchiolitis; however, its clinical significance requires further study.

\section{Background}

Bronchiolitis is the most common lower respiratory infection and one of the leading causes of hospitalization in young children ${ }^{1}$. Bronchiolitis is predominantly a viral disease caused by respiratory syncytial virus (RSV) in more than $70 \%$ of cases, followed by rhinovirus in descending order of frequency ${ }^{2}$. Despite the acute phase of bronchiolitis often requiring oxygen administration or admission into intensive care units, no effective treatment strategies have been established other than supportive care $^{1}$. In addition, an association between viral bronchiolitis and the development of recurrent wheezing and asthma as its long-term sequelae in later childhood has been demonstrated in various studies ${ }^{3,4}$. From the perspective of disease management, the prediction of disease severity and long-term prognosis may be significant in this disease.

To clarify the underlying immunological mechanisms and identify immune biomarkers predicting disease severity, numerous studies have evaluated immune biomarker concentrations with respect to disease severity in the blood of infants with bronchiolitis ${ }^{5}$. In a systematic review, less than two dozen biomarkers, including cytokines and chemokines, immune cell subsets, and gene variants, could significantly predict and stratify disease severity, but all such biomarkers had comparable studies that 
reported opposite results each other ${ }^{5}$, suggesting that there are no specific immune biomarkers of disease severity in infants with bronchiolitis.

Acid sphingomyelinase (ASM) is a key lysosomal enzyme in sphingolipid metabolism that hydrolyzes sphingomyelin, an important cellular membrane component, into ceramide and plays a role in normal membrane turnover ${ }^{6}$. On the other hand, ceramide functions as a second intracellular messenger implicated in a variety of cellular functions, and ASM activation has been reported as a proactive cellular response in many biological functions and pathological reactions in human conditions ${ }^{6}$. The sphingomyelin phosphodiesterase 1 gene that encodes ASM first leads to a common precursor protein, pre-pro-ASM, which is cleaved into pro-ASM and processed into the two types of ASM, lysosomal ASM (LASM) and S-ASM, through $\mathrm{N}$ - and C-terminal modifications and differential glycosylation inside Golgi ${ }^{7}$. LASM is transported into the lysosome, exposed to cellular pools of $\mathrm{Zn}^{2+}$, and then saturated with $\mathrm{Zn}^{2+}$ as a metallophosphodiesterase ${ }^{7}$. In contrast, S-ASM is secreted into the extracellular space and is located extracellularly without $\mathrm{Zn}^{2+}$ saturation ${ }^{7}$. Consequently, while L-ASM needs no additional $\mathrm{Zn}^{2+}$ ions for activation, S-ASM needs $\mathrm{Zn}^{2+}$ ions for activation and S-ASM activity can be measured using an assay buffer containing $\mathrm{Zn}^{2+}$ in various body fluids, including blood 8 ; ASM activation has been demonstrated to be linked with the extracellular secretion of ASM, which can be detected as an increase in S-ASM activity. However, the precise mechanisms of extracellular secretion of ASM remain to be elucidated.

More than 20 clinical studies have been conducted to measure the blood S-ASM activity in human diseases, resulting in a significant increase in S-ASM activity in various specific diseases, such as heart disease, diabetes mellitus 2, Alzheimer's disease, alcohol dependence, and inflammatory diseases including sepsis, systemic inflammatory response syndrome, hepatitis $C$, systemic vasculitis, inflammatory renal disease, and lymphohistiocytosis ${ }^{7}$. The results suggest that the increase in S-ASM activity might reflect cytokine release, inflammation, oxidative stress, or damage to the vascular endothelium, which is thought to be an abundant source of S-ASM ${ }^{7}$.

In our recent study, serum S-ASM activity was measured in children with acute common illness ${ }^{9}$. Fifty children, as a study subject, consisted of five groups based on clinical diagnoses: RSV bronchiolitis, adenovirus infection, streptococcal infection, asthma, and other infections due to unknown origin. As a result, the serum S-ASM activity was significantly elevated in a group of six patients with RSV bronchiolitis at more than two times that in healthy children and the other illness groups. The study preliminarily suggested an association between ASM activation and RSV bronchiolitis in infants.

In this study, serum S-ASM activity was studied in another cohort of 31 patients with RSV bronchiolitis to reveal the clinical significance of serum S-ASM activity.

\section{Materials And Methods}

\section{Study population}


Thirty-one infants, aged 0-11 months, diagnosed with RSV bronchiolitis were enrolled in this study. None of them were born prematurely (at or before 35 weeks of gestation) or had any history of diseases, including bronchopulmonary dysplasia, hemodynamically significant congenital heart diseases, neuromuscular disorders, immunocompromise, or Down syndrome. They had never received palivizumab injection before admission. The patients' demographic characteristics are presented in Table 1. They were admitted to the Akita Kousei Medical Center between August 2018 and January 2020, and their diagnosis of RSV infection was made performing antigen detection tests on nasopharyngeal discharges (ImunoAce RSV Neo, Shizuoka, Japan). The clinical diagnosis of RSV bronchiolitis was made based on criteria that comprised cough, increase in respiratory rate, chest retraction, prolongation of expiratory time, sibilant rhonchi, and hyperinflated lungs on chest $\mathrm{X}$-ray ${ }^{10}$. Blood was collected on admission and serum was stored frozen at $<-20^{\circ} \mathrm{C}$.

Eight healthy infants and the nine other RSV-negative hospitalized febrile infants, including three diagnoses of acute bronchitis, two of adenovirus infections, one of hand-foot-and-mouth disease, one of febrile convulsion, one of mycoplasma infection, and one of otitis media, all of whom presented with fever and respiratory symptoms, were also included as controls in this study. RSV-negative infections were confirmed using an antigen detection test.

Patients with RSV bronchiolitis were scored from 0 to 3 points for each of four factors (oxygen saturation of peripheral artery, respiratory rate, wheezing, and depressed breathing) for a maximum of 12 points, every day during hospitalization. We modified a previously reported scoring system (Table 2$)^{11,12}$. Medical records were accessed for age, sex, clinical symptoms, complications, need for oxygen administration, and laboratory data including white blood cell count, platelet count, aspartate aminotransferase, alanine aminotransferase, lactate dehydrogenase, hemoglobin, and C-reactive protein (CRP) levels.

\section{Determination of serum S-ASM activity, interferon-y (IFN-y), and interleukin-18 (IL-18)}

Serum S-ASM activity was measured as previously described ${ }^{13}$. Briefly, serum S-ASM activities were assayed using a substrate of ${ }^{14} \mathrm{C}$-labeled sphingomyelin (PerkinElmer, MA, USA) and a buffer containing $\mathrm{Zn}^{2+}$ as $\mathrm{Zn}^{2+}$-dependent S-ASM. A standard $200-\mu \mathrm{L}$ assay mixture was made up of $100 \mu \mathrm{L}$ serum, $50 \mu \mathrm{L}$ assay buffer (1.0M sodium acetate, $\mathrm{pH} 5.0$ ) with $4 \%$ Triton $\mathrm{X}-100$ as a final concentration of $1 \%$, and $50 \mu \mathrm{L}$ of substrate $\left(20 \mathrm{nmol}\right.$ of ${ }^{14} \mathrm{C}$-labeled sphingomyelin; $\left.0.08 \mu \mathrm{Ci} / 20 \mathrm{nmol}\right)$ in $0.2 \%$ taurodeoxycholic acid and then incubated at $37^{\circ} \mathrm{C}$ for $6 \mathrm{~h}$. The reaction was stopped with $200 \mu \mathrm{L}$ ice-cold $30 \%$ trichloroacetate and $400 \mu \mathrm{L} 2.5 \%$ bovine serum albumin. The mixtures were briefly vortexed and allowed to settle for $5 \mathrm{~min}$ before centrifugation $(3,000 \mathrm{rpm}$ for $5 \mathrm{~min}$ ). The supernatant $(500 \mu)$ was carefully transferred to glass scintillation pre-filled with $4.5 \mathrm{~mL}$ of Clearsol II (Nakalai Tesque, Kyoto, Japan). Radioactivity was measured using a liquid scintillation counter LSC 950 (Aloka, Tokyo, Japan). 
Levels of serum INF-y and IL-18 were determined using the Human IFN gamma ELISA Kit (Thermo Fisher Scientific, Waltham, MA, USA) and Human IL-18 ELISA Kit (MBL Inc., Tokyo, Japan), respectively.

\section{Statistical Analysis}

Data were analyzed using the IBM SPSS statistics 26.0 software package, and the results are presented as the mean \pm standard deviation. The Kruskal-Wallis test was used to compare the mean differences between the two groups. Spearman's rank correlation coefficient was used to examine the correlation between serum S-ASM levels with the clinical study measures. Statistical significance was set at $p<0.05$.

\section{Results}

The patients with RSV bronchiolitis, patients with RSV-negative febrile infection, and healthy controls are summarized in Table 1. Healthy controls were comparable to patients with RSV bronchitis in terms of age, sex, gestational age, and birth weight.

\section{Levels of serum S-ASM, IFN-y, and IL-18 in acute RSV bronchiolitis (Fig. 1)}

The mean serum S-ASM activity was $9.5 \pm 5.4 \mathrm{nmol} / \mathrm{mL} / \mathrm{h}$ in the 31 patients with RSV bronchiolitis, whereas the nine patients with RSV-negative febrile infection and the eight healthy infants had mean levels of $4.3 \pm 1.9 \mathrm{nmol} / \mathrm{mL} / \mathrm{h}$ and $4.0 \pm 1.4 \mathrm{nmol} / \mathrm{mL} / \mathrm{h}$, respectively. The serum S-ASM activity was significantly higher among the patients with RSV bronchiolitis than in the patients with RSV-negative febrile infection $(p<0.005)$ and healthy controls $(p<0.005)$.

The mean serum INF- $\gamma$ was $8.8 \pm 11.4 \mathrm{pg} / \mathrm{mL}$ in the 31 patients with RSV bronchiolitis, whereas the nine patients with RSV-negative febrile infection and eight healthy infants had mean levels of $14.0 \pm 23.7$ $\mathrm{pg} / \mathrm{mL}$ and $0.0 \pm 0.0 \mathrm{pg} / \mathrm{mL}$, respectively. The serum IFN- $\gamma$ level was significantly higher among the patients with RSV bronchiolitis $(p<0.005)$ and among the patients with RSV-negative febrile infection $(p<0.05)$ than in the healthy controls.

The mean serum IL-18 was $431.3 \pm 276.3 \mathrm{pg} / \mathrm{mL}$ in the 31 patients with RSV bronchiolitis, whereas the nine patients with RSV-negative febrile infection and eight healthy infants had levels of $260.9 \pm 77.5$ $\mathrm{pg} / \mathrm{mL}$ and $240.8 \pm 137.8 \mathrm{pg} / \mathrm{mL}$, respectively. No significant difference in IL-18 levels was observed between the three groups of infants.

\section{Relationship between S-ASM activity and clinical severity score, laboratory findings, and clinical observatory findings in RSV bronchiolitis}


The relationship between S-ASM activity and clinical severity score on admission was not statistically significant) (Fig.2). Spearman's rho correlations between S-ASM activity and age, sex, clinical severity score on admission, days from onset of fever, oxygen saturation, demand for oxygen therapy, duration of administration, administration of antibiotics, administration of steroids, white blood cells, CRP, and IL-18 were not statistically significant (Table 3 ). Spearman's rho correlation between S-ASM activity and IFN- $\gamma$ (rho $=-0.448, p=0.012$ ) was statistically significant (Table 3 ).

\section{Discussion}

The serum S-ASM activity was significantly higher among 31 infants with RSV bronchiolitis than in controls, which included healthy controls and RSV-negative febrile patients. This result confirmed our previous study that found higher serum S-ASM activity in six hospitalized infants with RSV bronchiolitis than that in five patients with adenovirus infection, nine patients with streptococcal infection, six patients with asthma attacks, and 24 patients with other infections ${ }^{9}$. Both results collectively suggest that elevated serum S-ASM activity may be a characteristic pathological reaction in infants with RSV bronchiolitis. During the last two decades, blood S-ASM activity has been studied and shown to be elevated in various specific human diseases ${ }^{7}$; however, this is the first study confirming that elevation of serum S-ASM activity is a characteristic pathological reaction in the acute phase of viral infection.

There has been only one study describing the relationship between viral infection and elevation of serum S-ASM activity ${ }^{14}$. In this study, significantly higher S-ASM activity was observed in the serum of patients with chronic hepatitis $C$ virus than that in healthy individuals. Especially in chronic hepatitis $C$ infection, S-ASM activity correlated significantly with markers of hepatic injury and showed high discriminative power. They concluded that chronic hepatitis $C$ virus infection and non-alcoholic fatty liver disease induces upregulation of serum acid sphingomyelinase, which appears to be a novel biomarker in hepatopathies. In our patients with RSV bronchiolitis, none of them had any liver dysfunction related to hepatic injury; thus, elevation of serum S-ASM activity may originate from a different mechanism than that due to hepatitis $\mathrm{C}$ virus infection.

Until now, increased S-ASM activity has been related to the enhanced release of the enzyme and enhanced activation via oxidative stress, inflammation, cytokine release, or damage to the vascular endothelium, and was, therefore, regarded as a state marker of these conditions ${ }^{7}$. In addition, S-ASM was specifically demonstrated to be a cytokine-responsive enzyme that increases in activity upon stimulation with interleukin- $1 \beta$ or tumor necrosis factor-a $(T N F-a)^{15}$. As is well known, acute bronchiolitis caused by RSV triggers an inflammatory response with the production and release of several pro-inflammatory cytokines $^{5,16}$. The two common cytokines are TNF- $a$ and INF- ${ }^{17}$. In this study, we did not measure the levels of TNF-a in our patients, but numerous studies measured the levels of TNF-a in patients with RSV bronchiolitis, reporting various associations between TNF-a levels and bronchiolitis severity. Thus, the elevation of S-ASM may originate from the cellular reaction to the release of various cytokines in RSV bronchiolitis. 
In addition, the relationship between S-ASM activity and laboratory data and clinical findings, including clinical severity score, was statistically analyzed in this study. No positive correlation was found and only serum INF-y was found to be negatively correlated. INF-y has been shown to be one of the various cytokines released in patients with RSV bronchiolitis. Numerous reports have attempted to clarify how the level of IFN- $y$ is associated with clinical severity or clinical findings. However, comparable studies have reported conflicting results ${ }^{5,17}$. Therefore, we could not speculate on the clinical and pathological significance of the negative correlation between S-ASM and INF-y in our study. Further study of S-ASM activity might be needed to clarify the original site and mechanism of ASM secretion in lower respiratory tract infections.

\section{Conclusion}

The present study demonstrated that blood S-ASM was significantly elevated in infants with RSV bronchiolitis, suggesting an association between ASM activation and RSV bronchiolitis in infants. In addition, blood levels of S-ASM might be a possible biomarker for clinical utility; however, its clinical significance requires further study.

\section{Abbreviations}

RSV: Respiratory syncytial virus ; S-ASM: Secretory acid sphingomyelinase ; INF- $\gamma$ : Interferon- $\gamma$; ASM: Acid sphingomyelinase ; L-ASM: lysosomal ASM ; IL-18: interleukin-18 ; INF-a: interferon-a.

\section{Declarations}

\section{Ethics approval and consent to participate}

Ethical approval was obtained from the Ethics Committee of Akita Kousei Medical Center and Akita University, Graduate School of Medicine, Akita, Japan. Written informed consent was obtained from the parents or guardians of patients and controls in accordance with the Declaration of Helsinki.

\section{Consent for publication}

Not applicable.

\section{Availability of data and materials}

The datasets used and/or analyzed during the current study are available from the corresponding author on reasonable request.

\section{Competing interests}


The authors declare no conflict of interests.

\section{Funding}

This study was unfunded.

\section{Authors' contribution}

S.Y., A.N., and T.T. designed the study; S.Y., A.N., C.H., T.H., D.K., H.K., and S.T. collected the data; S.T., A.N., and T.T. analyzed the samples; S.Y., A.N., and T.T. contributed to data analysis and interpretation; and S.Y., A.N., and T.T. wrote the manuscript. All authors edited the manuscript and agreed with the content submitted.

\section{Acknowledgements}

The authors thank Ms. Harumi Sugawara and Ms. Maiko Ito for their excellent technical assistances.

\section{Authors' information}

${ }^{1}$ Department of Pediatrics, Akita University Graduate School of Medicine, Hondo 1-1-1, Akita, Akita, 0108543, Japan. ${ }^{2}$ Division of Pediatrics, Akita Kousei Medical Center, Akita, Akita. ${ }^{3}$ Division of Pediatrics, Akita Red Cross Hospital, Akita, Akita.

\section{References}

1. Chatterjee A, Mavunda K, Krilov LR. Current state of respiratory syncytial virus disease and management. Infect Dis Ther. 2021;10(Suppl 1):5-16.

2. Ralston SL, Lieberthal AS, Meissner HC, Alverson BK, Baley JE, Gadomski AM, et al. Clinical practice guideline: the diagnosis, management, and prevention of bronchiolitis. Pediatrics 2014;134(5):e1474502.

3. Knudson CJ, Varga SM. The relationship between respiratory syncytial virus and asthma. Vet Pathol 2015;52(1):97-106.

4. Zeng R, Li C, Li N, Wei L, Cui Y. The role of cytokines and chemokines in severe respiratory syncytial virus infection and subsequent asthma. Cytokine 2011;53(1):1-7.

5. Hancock DG, Cavallaro EC, Doecke E, Reynolds M, Charles-Britton B, Dixon D, et al. Immune biomarkers predicting bronchiolitis disease severity: A systematic review. Paediatr Respir Rev 2019;32:82-90. 
6. Schuchman EH. Acid sphingomyelinase, cell membranes and human disease: Lessons from Niemann-Pick disease. FEBS Lett 2010;584(9):1895-900.

7. Kornhuber J, Rhein C, Müller CP, Mühle C. Secretory sphingomyelinase in health and disease. Biol Chem 2015;396(6-7):707-36.

8. Takahashi I, Takahashi T, Abe T, Watanabe W, Takada G. Distribution of acid sphingomyelinase in human various body fluids. Tohoku J Exp Med 2000;192(1):61-6.

9. Yoshida S, Noguchi A, Kikuchi W, Fukaya H, Igarashi K, Takahashi T. Elevation of serum acid sphingomyelinase activity in children with acute respiratory syncytial virus bronchiolitis. Tohoku $\mathrm{J}$ Exp Med 2017;243(4):275-81.

10. Mehta R, Scheffler M, Tapia L, Aideyan L, Patel KD, Jewell AM, et al. Lactate dehydrogenase and caspase activity in nasopharyngeal secretions are predictors of bronchiolitis severity. Influ Other Respir Viruses 2014;8(6):617-25.

11. Tal A, Bavilski C, Yohai D, Bearman JE, Gorodischer R, Moses SW. Dexamethasone and salbutamol in the treatment of acute wheezing in infants. Pediatrics 1983;71(1):13-8.

12. De Boeck K, Van der Aa N, Van Lierde S, Corbeel L, Eeckels R. Respiratory syncytial virus bronchiolitis: A double-blind dexamethasone efficacy study. J Pediatr 1997;131(6):919-21.

13. Konno Y, Takahashi I, Narita A, Takeda O, Koizumi H, Tamura M, et al. Elevation of serum acid sphingomyelinase activity in acute Kawasaki disease. Tohoku J Exp Med 2015;237(2):133-40.

14. Grammatikos G, Mühle C, Ferreiros N, Schroeter S, Bogdanou D, Schwalm S, et al. Serum acid sphingomyelinase is upregulated in chronic hepatitis $\mathrm{C}$ infection and non alcoholic fatty liver disease. Biochim Biophys Acta 2014;1841(7):1012-20.

15. Marathe S, Schissel SL, Yellin MJ, Beatini N, Mintzer R, Williams KJ, et al. Human vascular endothelial cells are rich and regulatable source of secretory sphingomyelinase. Implication for early atherogenesis and ceramide-mediated cell signaling. J Biol Chem 1998;273(7):4081-8.

16. Vázquez Y, González L, Noguera L, González PA, Riedel CA, Bertrand P, et al. Cytokines in the respiratory airway as biomarkers of severity and prognosis for respiratory syncytial virus infection: An update. Front Immunol 2019;10:1154.

17. Akhilesh K, Nilanjana D, Himanshu K. COV ert ID entities of current worldwide pandemic. Int Rev Immunol 2021;40(1-2):1-4.

\section{Tables}

Due to technical limitations, tables are only available as a download in the Supplemental Files section.

\section{Figures}


Fig.1

$* \mathbf{p}<0.05$

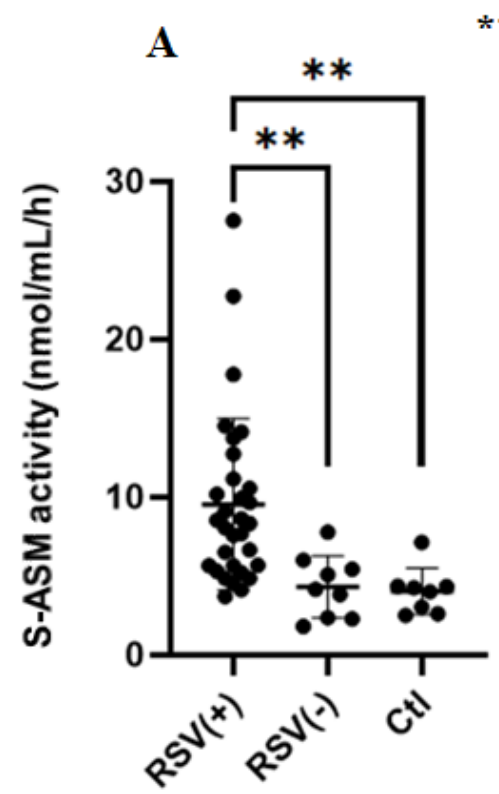

$* * \mathbf{p}<0.005$
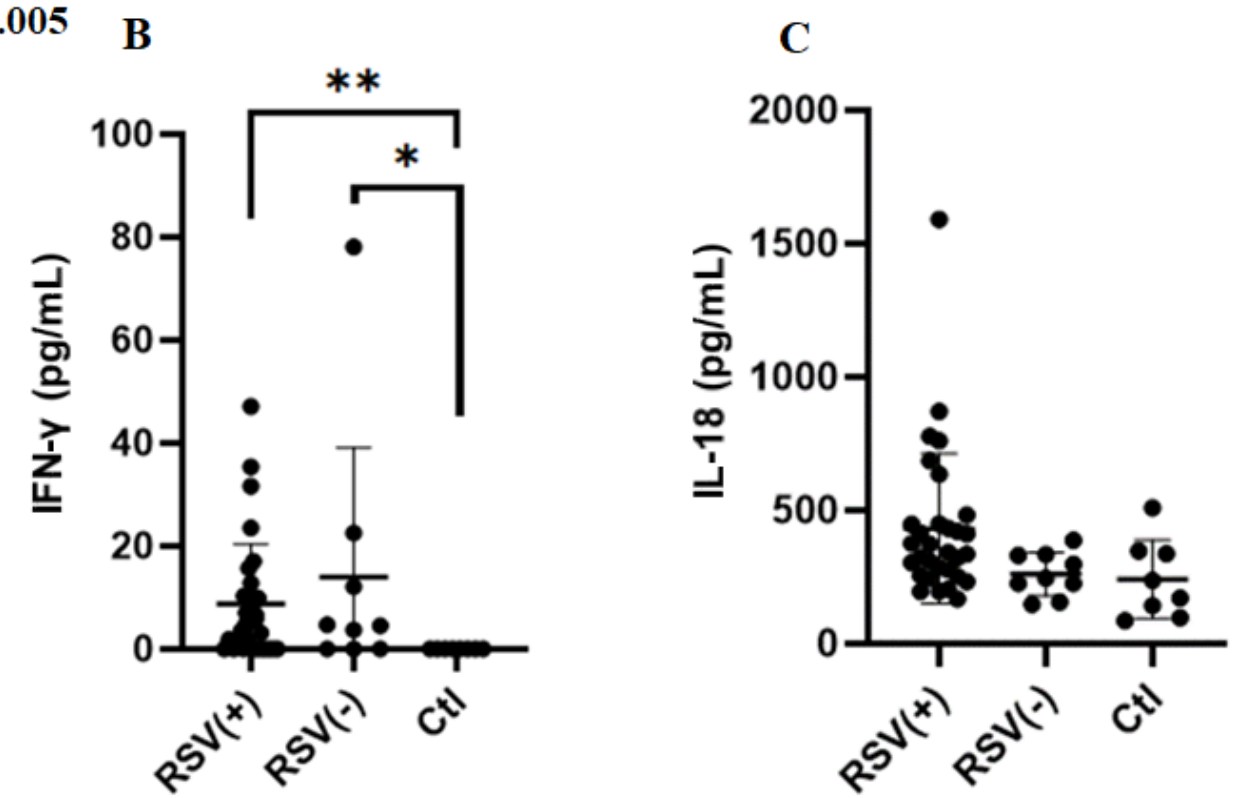

Figure 1

S-ASM activity, and INF-y and IL-18 levels in acute RSV infection. Serum S-ASM activity (A), IFN-y levels (B), and IL-18 levels (C) are shown for the RSV(+), RSV(-), and Control (CtI) groups.

Fig.2

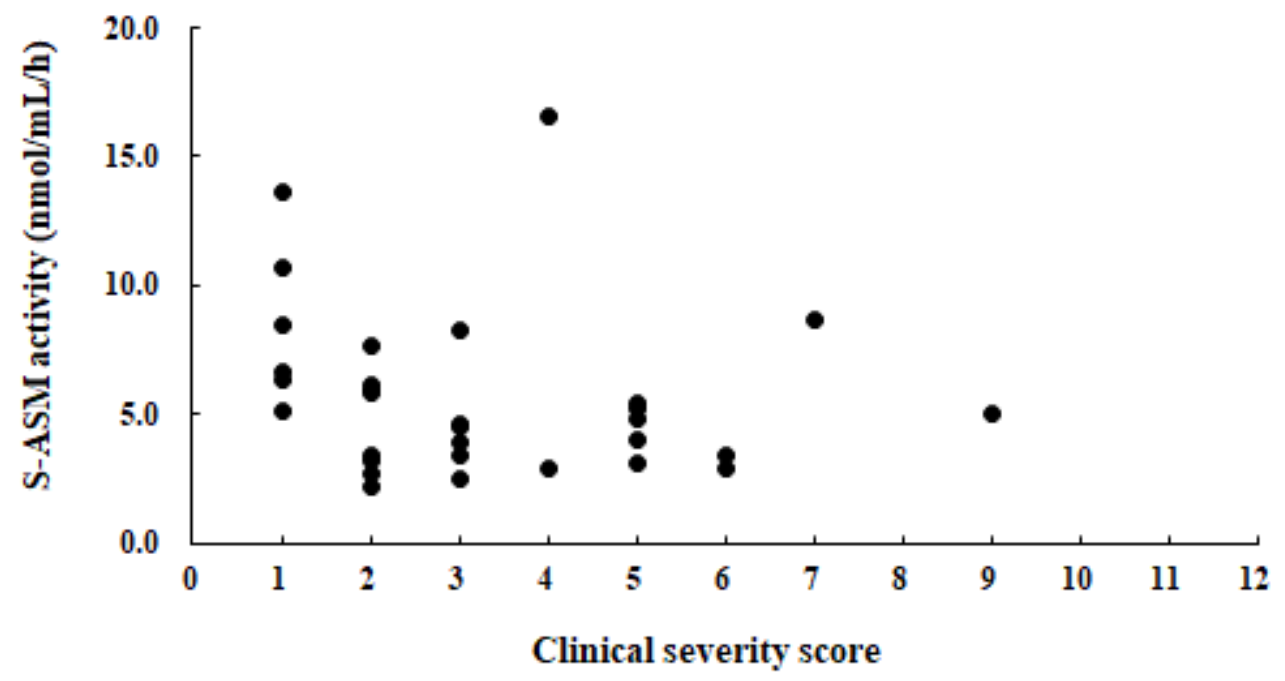

Figure 2 
Serum S-ASM activity and clinical severity score on admission in infants with RSV bronchiolitis.

\section{Supplementary Files}

This is a list of supplementary files associated with this preprint. Click to download.

- Table1YamamotoAkitatoBMCPediatr2.docx

- Table2yamamotoAkitatoBMCPediatr2.docx

- Table3YamamotoAKitatoBMCPediatrics2.docx 